\title{
Cluster solutions for the Schrödinger-Poisson-Slater problem around a local minimum of the potential
}

\section{David Ruiz and Giusi Vaira}

\begin{abstract}
In this paper we consider the system in $\mathbb{R}^{3}$

$$
\left\{\begin{array}{l}
-\varepsilon^{2} \Delta u+V(x) u+\phi(x) u=u^{p} \\
-\Delta \phi=u^{2}
\end{array}\right.
$$

for $p \in(1,5)$. We prove the existence of multi-bump solutions whose bumps concentrate around a local minimum of the potential $V(x)$. We point out that such solutions do not exist in the framework of the usual Nonlinear Schrödinger Equation.
\end{abstract}

\section{Introduction and main results}

Recently, many papers have studied different versions of the SchrödingerPoisson- $X^{\alpha}$ problem:

$$
-\frac{\hbar^{2}}{2 m} \Delta u+V(x) u+\left(u^{2} \star \frac{1}{4 \pi|x|}\right) u=|u|^{p-1} u, x \in \mathbb{R}^{3},
$$

where $V(x)$ is an external potential and $p \in(1,5)$. The interest on this problem stems from the Slater approximation of the exchange term in the Hartree-Fock model, see [24]. In this framework, $p=5 / 3$; however, other exponents have been used in different approximations, which have been referred to as $X^{\alpha}$ type approximations, see [21]. From another point of view, this equation has been proposed in [5] under the name of SchrödingerMaxwell equation. For more information on the relevance of this model and its deduction, we refer to $[5,6,7,8,21,25]$.

2000 Mathematics Subject Classification: 35B40, 35J20, 35J55.

Keywords: Nonlinear Analysis, Schrödinger-Poisson-Slater problem, variational methods, singular perturbation method, multi-bump solutions. 
From the mathematical point of view, problem (1.1) presents an interaction between two different kind of nonlinear terms: a repulsive nonlocal term and an attractive local term. This, and related problems, have been much studied recently by using variational methods, see $[3,4,9,10,16,17,22,23$, $26,27]$.

If we define $\phi_{u}=u^{2} \star \frac{1}{4 \pi|x|}$ and $\varepsilon^{2}=\frac{\hbar^{2}}{2 m}$, the equation (1.1) can be rewritten as a system in the form:

$$
\left\{\begin{array}{l}
-\varepsilon^{2} \Delta u+V(x) u+\phi(x) u=|u|^{p-1} u \\
-\Delta \phi=u^{2}
\end{array}\right.
$$

In this paper we are concerned with the semiclassical limit for the system (1.2), namely the problem of finding non trivial solutions $(u, \phi) \in$ $H^{1}\left(\mathbb{R}^{3}\right) \times D^{1,2}\left(\mathbb{R}^{3}\right)$ and studying their asymptotic behavior as $\varepsilon \rightarrow 0$. Such solutions are usually referred to as semiclassical states.

A large number of papers study the semiclassical states for the following nonlinear Schrödinger equation

$$
-\varepsilon^{2} \Delta u+V(x) u=|u|^{p-1} u, \quad x \in \mathbb{R}^{3} .
$$

For the problem (1.3) spike solutions are found around the critical points of the potential $V$, see for instance $[1,20]$. These are solutions that concentrate (as $\varepsilon \rightarrow 0$ ) around a unique point, and tend to zero uniformly outside a ball centered at this point. For instance in [20] Yanyan Li proved the existence of positive solutions concentrating near $C^{1}$ stable critical points of $V$. Moreover, Li proves also the existence of multi-bump solutions, namely, solutions concentrating around different critical points of $V$. Other results in this direction were given in $[13,14]$. However, in the previous papers the bumps are well separated and so the interactions among the different bumps are neglected.

In [15] the authors prove the existence of multi-bump solutions for (1.3) whose bumps tend to a point of local maximum of $V$. Here the interactions among the bumps do play a role. In a certain sense, each bump has an attractive effect on the other bumps, whereas the potential has a repulsive effect (around its local maximum). The multi-bump solution exists due to a balance between the two effects. The authors also show that multi-bump solutions do not exist around nondegenerate local minima. In this case, both effects would be attractive and no balance could be possible.

With respect to (1.2), the existence of single-bump solutions near critical points of $V$ has been recently proved, see [19]. Other concentration phenomena have been proved for this system even with the absence of the potential, see $[11,12]$. 
In this paper we prove the existence of positive solutions with $K$ interacting bumps around local minima of the potential $V$. These solutions appear because of the effect of the Poisson term in our equation. Indeed, the Poisson term implies a repulsive effect among the bumps which balance the attractive effect of the potential $V$.

We assume that:

(V1) $V$ has a local strict minimum point in $P_{0}$, namely there exists a bounded open set $\mathcal{U}$ such that $P_{0} \in \mathcal{U}$ and

$$
V\left(P_{0}\right)=\min _{x \in \overline{\mathcal{U}}} V(x)<V(P), \quad \forall P \in \mathcal{U} \backslash\left\{P_{0}\right\}
$$

Up to a translation and dilatation, we can assume $P_{0}=0, V(0)=1$.

$(\mathrm{V} 2) V(x)=1+|g(x)|^{\alpha}$ for any $x \in \mathcal{U}$, where $g: \mathcal{U} \rightarrow \mathbb{R}$ is a $C^{2,1}$ function and $\alpha>2$.

In particular, there holds:

(V2') $V(x) \leq 1+C|x|^{\alpha}$ for $x \in \mathcal{U}$ and some $C>0$.

(V3) $\inf V>0$.

Observe that under the above conditions the local minimum must be degenerate. We point out that conditions (V1)-(V2')-(V3) are sufficient for most of our arguments. We need condition (V2) for technical reasons, to be able to rule out possible undesired oscillations of the derivatives of $V$ near 0 .

Let us denote by $U$ the unique positive radial solution in $H^{1}\left(\mathbb{R}^{3}\right)$ of the problem (see [18]):

$$
-\Delta U+U=U^{p}
$$

Our main result is the following.

Theorem 1.1. Assume that $V$ satisfies (V1), (V2) and (V3) and suppose $p \in(1,5)$. Then for any positive integer $K \in \mathbb{Z}$, there exists $\varepsilon_{K}>0$ such that for any $\varepsilon<\varepsilon_{K}$ there exists a positive solution $u_{\varepsilon}$ of (1.2) with $K$ bumps converging to 0 . More specifically, there exists $Q_{1}^{\varepsilon}, \ldots Q_{k}^{\varepsilon} \in \mathbb{R}^{3}$ such that:

1. $Q_{i}^{\varepsilon} \rightarrow 0, \varepsilon^{-1}\left|Q_{i}^{\varepsilon}\right| \rightarrow+\infty$ as $\varepsilon \rightarrow 0$.

2. Defining $\tilde{u}_{\varepsilon}(x)=u_{\varepsilon}(\varepsilon x)$, we have that $\tilde{u}_{\varepsilon}(x)=\sum_{i=1}^{K} U\left(x-\varepsilon^{-1} Q_{i}^{\varepsilon}\right)+$ $o(1)$, as $\varepsilon \rightarrow 0$.

The proof uses a singular perturbation method, based on a LyapunovSchmidt reduction. We point out that the distance between the bumps is different from that of the multi-bump solutions of [15], and this is caused because the different balance involving the Poisson term. 
The paper is organized as follows. Section 2 is devoted to some notations and to the variational setting of the problem. In Section 3 we introduce the Lyapunov-Schmidt reduction and solve the auxiliary equation. Finally, in Section 4 the reduced functional is studied, solving the bifurcation equation. This completes the proof of Theorem 1.1.

\section{Preliminaries}

As mentioned in the introduction, we denote by $U$ the unique positive radial solution in $H^{1}\left(\mathbb{R}^{3}\right)$ of the problem

$$
-\Delta U+U=U^{p}
$$

This solution satisfies the following decay property (see [18]):

$$
\lim _{r \rightarrow+\infty} U(r) r e^{r}=C>0, \quad \lim _{r \rightarrow+\infty} \frac{U^{\prime}(r)}{U(r)}=-1, \quad r=|x| .
$$

for some constant $C$.

The function $U$ is a critical point of the $C^{2}$ functional $I_{0}: H^{1}\left(\mathbb{R}^{3}\right) \rightarrow \mathbb{R}$ defined as

$$
I_{0}(u)=\frac{1}{2}\|u\|^{2}-\frac{1}{p+1} \int_{\mathbb{R}^{3}}|u|^{p+1} d x,
$$

where $\|\cdot\|$ denotes the usual norm in $H^{1}\left(\mathbb{R}^{3}\right)$. Furthermore the solution $U$ is nondegenerate (up to translations). More specifically, there holds:

Lemma 2.1. Define the operator $Q: H^{1}\left(\mathbb{R}^{3}\right) \rightarrow \mathbb{R}$ as

$$
Q[\nu]:=I_{0}^{\prime \prime}(U)[\nu, \nu]=\int_{\mathbb{R}^{3}}\left[|\nabla \nu|^{2}+\nu^{2}-p U^{p-1} \nu^{2}\right] d x .
$$

We denote $U_{k}=\frac{\partial U}{\partial x_{k}}$. Then there hold:

- $Q[U]=(1-p)\|U\|^{2}<0$.

- $Q\left[\frac{\partial U}{\partial x_{j}}\right]=0, j=1,2,3$.

- $Q[\nu] \geq C\|\nu\|^{2}$ for all $\nu \perp U, \nu \perp \frac{\partial U}{\partial x_{j}}, j=1,2,3$.

For a proof see for instance [2, Lemma 8.6].

It is convenient to make the change of variable $x \mapsto \varepsilon x$ and so we arrive to the problem:

$$
-\Delta u+V(\varepsilon x) u+\varepsilon^{2} \phi_{u} u=u^{p}, \quad u \in H^{1}\left(\mathbb{R}^{3}\right), \quad u>0 .
$$

Here $\phi_{u} \in D^{1,2}\left(\mathbb{R}^{3}\right)$, and

$$
\int_{\mathbb{R}^{3}}\left|\nabla \phi_{u}\right|^{2} d x=\int_{\mathbb{R}^{3}} \phi_{u} u^{2} d x=\int_{\mathbb{R}^{3}} \int_{\mathbb{R}^{3}} \frac{u^{2}(x) u^{2}(y)}{4 \pi|x-y|} d x d y .
$$


In general, given $f \in L^{6 / 5}$, the solution of the problem $-\Delta \phi=f$ belongs to $D^{1,2}\left(\mathbb{R}^{3}\right)$ and:

$$
\int_{\mathbb{R}^{3}} \nabla \phi \cdot \nabla \psi=\int_{\mathbb{R}^{3}} f \psi \leq\|\psi\|_{L^{6}}\|f\|_{L^{6 / 5}} \leq C\|\psi\|_{D^{1,2}}\|f\|_{L^{6 / 5}}
$$

Therefore, $\|\phi\|_{D^{1,2}} \leq C\|f\|_{6 / 5}$.

Moreover, it is well-known (see [5], for example) that the solutions of (2.2) correspond to positive critical points of the $C^{2}$ functional $I_{\varepsilon}: H^{1}\left(\mathbb{R}^{3}\right) \rightarrow \mathbb{R}$,

$$
\begin{aligned}
& I_{\varepsilon}(u)= \\
= & \frac{1}{2} \int_{\mathbb{R}^{3}}\left[|\nabla u|^{2}+V(\varepsilon x) u^{2}\right] d x+\frac{\varepsilon^{2}}{4} \int_{\mathbb{R}^{3}} \phi_{u}(x) u^{2} d x-\frac{1}{p+1} \int_{\mathbb{R}^{3}}|u|^{p+1} d x .
\end{aligned}
$$

Finally, let us compute the derivatives of $V$. By using (V2)

$$
\begin{aligned}
& V_{x_{i}}(x)=\alpha|g(x)|^{\alpha-2} g(x) g_{x_{i}}(x), \\
& V_{x_{i} x_{j}}(x)=\alpha(\alpha-1)|g(x)|^{\alpha-2} g_{x_{i}}(x) g_{x_{j}}(x)+\alpha|g(x)|^{\alpha-2} g(x) g_{x_{i} x_{j}}(x) .
\end{aligned}
$$

In particular $V \in C^{2, \gamma}(\mathcal{U})$, where $\gamma=\min \{1, \alpha-2\}$.

\section{The Lyapunov-Schmidt reduction. The auxiliary equ- ation}

In this section we begin the Lyapunov-Schmidt for the proof of Theorem 1.1. This will be made around an appropriate set of "approximating solutions". For any $K \in \mathbb{N}$, we define

$\Lambda_{\varepsilon}=\left\{\mathbf{P} \in \mathbb{R}^{3 K}:\left|P_{i}-P_{j}\right| \geq \varepsilon^{\frac{2-\alpha}{\alpha+1}+\delta}, i \neq j, V\left(\varepsilon P_{i}\right) \leq 1+\varepsilon^{\frac{3 \alpha}{\alpha+1}-\delta}, \varepsilon P_{i} \in \mathcal{U}\right\}$

where $\delta>0$ is chosen small enough so that $\frac{3 \alpha}{\alpha+1}-\delta>2$ (this is possible since $\alpha>2$ ). Observe that $\frac{2-\alpha}{\alpha+1}+\delta<0$ and $\Lambda_{\varepsilon}$ is not empty for $\varepsilon$ small enough.

Fix $\mathbf{P}=\left(P_{1}, \ldots, P_{K}\right) \in \Lambda_{\varepsilon}$. Setting $z_{P_{i}}(x)=U\left(x-P_{i}\right)$, we define the manifold of "approximate solutions":

$$
\mathcal{Z}=\left\{z_{\mathbf{P}}(x)=\sum_{i=1}^{K} z_{P_{i}}(x): \quad \mathbf{P} \in \Lambda_{\varepsilon}\right\} .
$$

This section is devoted to the proof of the next result:

Proposition 3.1. Assume that $V$ satisfies (V1), (V2) and (V3) and suppose $p \in(1,5)$. Then for any positive integer $K \in \mathbb{Z}$, there exists $\varepsilon_{K}>0$ such that for any $\varepsilon<\varepsilon_{K}$ there exists a positive solution $u_{\varepsilon}$ of (2.2), and $z_{\varepsilon} \in \mathcal{Z}$ such that $\left\|u_{\varepsilon}-z_{\varepsilon}\right\|=O\left(\varepsilon^{2}\right)$. 
It is easy to check that Proposition 3.1 implies Theorem 1.1.

The proof uses a Lyapunov-Schmidt reduction. For every $z \in \mathcal{Z}$, we define $W=W_{z, \varepsilon}=\left(T_{z} \mathcal{Z}\right)^{\perp}$ and $P: H^{1}\left(\mathbb{R}^{3}\right) \rightarrow W$ the orthogonal projection onto $W$. Our approach is to find a pair $z \in \mathcal{Z}, w \in W,\|w\|=O\left(\varepsilon^{2}\right)$, such that $I_{\varepsilon}^{\prime}(z+w)=0$. Equivalently:

$$
\left\{\begin{array}{l}
\text { a) } P I_{\varepsilon}^{\prime}(z+w)=0 \\
\text { b) }(\mathcal{I}-P) I_{\varepsilon}^{\prime}(z+w)=0
\end{array}\right.
$$

The first equation above is called auxiliary equation, and the second one receives the name of bifurcation equation.

Our intention now is to find a solution $w \in W$ of the auxiliary equation for any $z \in \mathcal{Z}$. We begin with some estimates:

Proposition 3.2. There exists $C=C(K)>0$ such that for all $\varepsilon>0$ small and any $\mathbf{P} \in \Lambda_{\varepsilon}$, we have

$$
\left\|I_{\varepsilon}^{\prime}\left(z_{\mathbf{P}}\right)\right\| \leq C \varepsilon^{2}
$$

Proof. Taking into account that $z_{P_{i}}$ are solutions of (1.4), we have:

$$
\begin{aligned}
I_{\varepsilon}^{\prime}\left(z_{\mathbf{P}}\right)[v]=\underbrace{\int_{\mathbb{R}^{3}}[V(\varepsilon x)-1] z_{\mathbf{P}} v d x}_{(I)} & +\underbrace{\varepsilon^{2} \int_{\mathbb{R}^{3}} \phi_{z_{\mathbf{P}}} z_{\mathbf{P}} v d x}_{(I I)} \\
& -\underbrace{\int_{\mathbb{R}^{3}}\left[\left|z_{\mathbf{P}}\right|^{p}-\sum_{i=1}^{K} z_{P_{i}}^{p}\right] v d x}_{(I I I)}
\end{aligned}
$$

Let us evaluate separately the various terms. The second term can be easily estimated (see Section 2):

$$
(I I) \leq\left\|z_{\mathbf{P}}\right\|^{3} \cdot\|v\| \leq C K\|v\| .
$$

For (I), it suffices to estimate

$$
\int_{\mathbb{R}^{3}}[V(\varepsilon x)-1] z_{P_{i}} v d x \leq \underbrace{\int_{\mathbb{R}^{3}}\left[V(\varepsilon x)-V\left(\varepsilon P_{i}\right)\right] z_{P_{i}} v d x}_{(A)}+\underbrace{\int_{\mathbb{R}^{3}}\left[V\left(\varepsilon P_{i}\right)-1\right] z_{P_{i}} v d x}_{(B)} .
$$

By the definition of $\Lambda_{\varepsilon}$, we get that $(B)=o\left(\varepsilon^{2}\right)$. Let us estimate $(A)$ by splitting the integral in two parts:

$$
\begin{aligned}
\int_{\mathbb{R}^{3}}\left[V(\varepsilon x)-V\left(\varepsilon P_{i}\right)\right] z_{P_{i}} v d x= & \int_{\left|x-P_{i}\right|>\varepsilon^{-1}}\left[V(\varepsilon x)-V\left(\varepsilon P_{i}\right)\right] z_{P_{i}} v d x \\
& +\int_{\left|x-P_{i}\right|<\varepsilon^{-1}}\left[V(\varepsilon x)-V\left(\varepsilon P_{i}\right)\right] z_{P_{i}} v d x .
\end{aligned}
$$


Since $V$ is bounded in $L^{\infty}$, we use Hölder estimate and the change $y=x-P_{i}$, to conclude

$\int_{\left|x-P_{i}\right|>\varepsilon^{-1}}\left[V(\varepsilon x)-V\left(\varepsilon P_{i}\right)\right] z_{P_{i}} v d x \leq C\left(\int_{|y|>\varepsilon^{-1}} U^{2}(y) d y\right)^{1 / 2}\|v\|_{L^{2}}=o\left(\varepsilon^{M}\right)\|v\|_{L^{2}}$

for any $M>0$, thanks to the exponential decay of $U$.

Observe that if $\left|x-P_{i}\right|<\varepsilon^{-1}, \varepsilon P_{i}$ belongs to $\mathcal{U}$ and $d(\varepsilon x, \mathcal{U}) \leq 1$. We use a Taylor expansion:

$$
\begin{aligned}
\int_{\left|x-P_{i}\right|<\varepsilon^{-1}} \mid & V(\varepsilon x)-V\left(\varepsilon P_{i}\right)\left|z_{P_{i}}\right| v \mid d x \leq \\
\leq & \int_{\mathbb{R}^{3}}\left(\varepsilon\left|\nabla V\left(\varepsilon P_{i}\right)\right|\left|x-P_{i}\right|+C \varepsilon^{2}\left|x-P_{i}\right|^{2}\right) z_{P_{i}}|v| d x .
\end{aligned}
$$

Again by the exponential decay of $U,\left\|\left|x-P_{i}\right|^{m} z_{P_{i}}\right\|_{L^{2}}$ is uniformly bounded for any $m>0$. So it suffices to estimate $\left|\nabla V\left(\varepsilon P_{i}\right)\right|$.

Recall that $\varepsilon P_{i} \in \Lambda_{\varepsilon}$, and so $V\left(\varepsilon P_{i}\right)=1+\left|g\left(\varepsilon P_{i}\right)\right|^{\alpha} \leq 1+\varepsilon^{\frac{3 \alpha}{\alpha+1}-\delta}$. By (2.4),

$$
\left|V_{x_{i}}(x)\right| \leq C|g(x)|^{\alpha-1} \leq C \varepsilon^{\left(\frac{3 \alpha}{\alpha+1}-\delta\right) \frac{\alpha-1}{\alpha}} .
$$

Observe that $\frac{3 \alpha}{\alpha+1}-\delta>2>\frac{\alpha}{\alpha-1}$. Therefore, $\nabla V\left(\varepsilon P_{i}\right)=o(\varepsilon)$.

Finally we consider (III). These estimates have been done in [15]; we sketch here the proof for the sake of completeness. Let us define $\rho_{\varepsilon}=\varepsilon^{\frac{2-\alpha}{\alpha+1}+\delta}$ and divide $\mathbb{R}^{3}$ in $K+1$ regions:

$$
\Omega_{i}=\left\{x \in \mathbb{R}^{3}: 2\left|x-P_{i}\right| \leq \rho_{\varepsilon}\right\} \quad \text { for } i=1 \ldots K, \quad \Omega_{0}=\mathbb{R}^{3} \backslash\left(\cup_{i=1}^{K} \Omega_{i}\right) .
$$

We now use the $C^{1, \sigma}$ regularity of the function $f(u)=u^{p}$, where $\sigma=$ $\min \{1, p-1\}$ :

$$
\begin{aligned}
\int_{\Omega_{j}} \mid & \left(\sum_{i=1}^{K} z_{P_{i}}\right)^{p}-z_{P_{j}}^{p}-\sum_{i \neq j} z_{P_{i}}^{p}|| v \mid d x \\
& \leq \int_{\Omega_{j}}\left[p z_{P_{j}}^{p-1}\left(\sum_{i \neq j} z_{P_{i}}\right)+C\left(\sum_{i \neq j} z_{P_{i}}\right)^{1+\sigma}+\sum_{i \neq j} z_{P_{i}}^{p}\right]|v| d x \\
& \leq C \int_{\Omega_{j}}\left(\sum_{i \neq j} z_{P_{i}}\right)|v| d x .
\end{aligned}
$$

The last inequality is due to the fact that in $\Omega_{j}, z_{P_{i}} \leq 1$. Indeed, defining $\rho_{\varepsilon}=\varepsilon^{\frac{2-\alpha}{\alpha+1}+\delta}$ and using the exponential decay of $U$, we have

$$
\int_{\Omega_{j}} z_{P_{i}}^{2}(x) d x \leq \int_{2|x|>\rho_{\varepsilon}} U^{2}(y) d y \leq C \int_{2 r>\rho_{\varepsilon}} e^{-2 y} d r=C e^{-\rho_{\varepsilon}} .
$$


On the other hand,

$$
\begin{gathered}
\int_{\Omega_{0}}\left|\left(\sum_{i=1}^{K} z_{P_{i}}\right)^{p}-\sum_{i=1}^{K} z_{P_{i}}^{p}\right||v| \leq C \int_{\Omega_{0}} \sum_{i=1}^{K} z_{P_{i}}^{p}|v|, \\
\int_{\Omega_{0}} z_{P_{i}}^{2 p}(x) d x \leq \int_{2|x|>\rho_{\varepsilon}} U^{2 p}(y) d y \leq C e^{-p \rho_{\varepsilon}} .
\end{gathered}
$$

This concludes the estimate (III).

Now we are concerned with the invertibility of $I_{\varepsilon}^{\prime \prime}\left(z_{\mathbf{P}}\right)$ on $W=\left(T_{z_{\mathbf{P}}}(\mathcal{Z})\right)^{\perp}$. First we observe that $T_{z_{\mathbf{P}}} \mathcal{Z}$ is spanned by the functions $\dot{z}_{i, j}:=\frac{\partial U}{\partial x_{j}}\left(x-P_{i}\right)$, with $i=1, \ldots, K$ and $j=1,2,3$. Recall that $P$ denotes the orthogonal projection onto $W$; me decompose: $W=A \oplus B$ where

$$
A=\left\langle\left\{P z_{P_{i}}\right\}_{i=1 \ldots K}\right\rangle \text { and } B=\left(A \oplus T_{z_{\mathbf{P}}} \mathcal{Z}\right)^{\perp}
$$

Proposition 3.3. For $\varepsilon$ small and any $\mathbf{P} \in \Lambda_{\varepsilon}, P I_{\varepsilon}^{\prime \prime}\left(z_{\mathbf{P}}\right): W \rightarrow W$ is invertible and $\left\|\left[P I_{\varepsilon}^{\prime \prime}\left(z_{\mathbf{P}}\right)\right]^{-1}\right\| \leq \bar{C}$.

The above result follows directly from the following lemma (see [2]):

Lemma 3.4. For all $\varepsilon>0$ sufficiently small there exist two positive constants $C_{1}, C_{2}$ such that

(a) $I_{\varepsilon}^{\prime \prime}\left(z_{\mathbf{P}}\right)[u, u] \leq-C_{1}\|u\|^{2}$, for all $u \in A$;

(b) $I_{\varepsilon}^{\prime \prime}\left(z_{\mathbf{P}}\right)[u, u] \geq C_{2}\|u\|^{2}$, for all $u \in B$.

Proof. Let be $u \in A$. Then

$$
u=\sum_{i=1}^{K} \lambda_{i} P z_{P_{i}}, \quad \lambda_{i} \in \mathbb{R}, \quad i=1, \ldots, K .
$$

For $i=1, \ldots, K, P z_{P_{i}}$ are orthogonal to $T_{z_{\mathbf{P}}}(\mathcal{Z})$. Hence we can write

$$
P z_{P_{i}}=z_{P_{i}}-\psi_{i}, \quad i=1, \ldots, K
$$

where $\psi_{i}$ are given by

$$
\psi_{i}=\sum_{\substack{l, j \\ l \neq i}}\left(z_{P_{i}}, \dot{z}_{l, j}\right) \frac{\dot{z}_{l, j}}{\left\|\dot{z}_{l, j}\right\|^{2}}
$$

The functions $\dot{z}_{l, j}$ satisfy $-\Delta \dot{z}_{l, j}+\dot{z}_{l, j}=p z_{P_{l}}^{p-1} \dot{z}_{l, j}$. Since for $i \neq l,\left|P_{i}-P_{l}\right| \rightarrow$ $+\infty$ as $\varepsilon \rightarrow 0$, after an integration by parts, we get $\left(z_{P_{i}}, \dot{z}_{l, j}\right)=o(1)$ as $\varepsilon \rightarrow 0$. This implies $\left\|\psi_{i}\right\|=o(1)$ as $\varepsilon \rightarrow 0$ for $i=1, \ldots, K$. 
We now apply the bilinear form given by $I_{\varepsilon}^{\prime \prime}\left(z_{\mathbf{P}}\right)$ to obtain

$$
\begin{aligned}
I_{\varepsilon}^{\prime \prime}\left(z_{\mathbf{P}}\right)[u, u]= & \underbrace{I_{\varepsilon}^{\prime \prime}\left(z_{\mathbf{P}}\right)\left[\sum_{i=1}^{K} \lambda_{i} z_{P_{i}}, \sum_{i=1}^{K} \lambda_{i} z_{P_{i}}\right]}_{(I)}+\underbrace{I_{\varepsilon}^{\prime \prime}\left(z_{\mathbf{P}}\right)\left[\sum_{i=1}^{K} \lambda_{i} \psi_{i}, \sum_{i=1}^{K} \lambda_{i} \psi_{i}\right]}_{(I I)} \\
& +\underbrace{2 I_{\varepsilon}^{\prime \prime}\left(z_{\mathbf{P}}\right)\left[\sum_{i=1}^{K} \lambda_{i} z_{P_{i}} \sum_{i=1}^{K} \lambda_{i} \psi_{i}\right]}_{(I I I)} .
\end{aligned}
$$

We observe that $I_{\varepsilon}^{\prime \prime}\left(z_{\mathbf{P}}\right)$ maps bounded sets onto bounded sets, then since $z_{\mathbf{P}}$ is bounded

$$
(I I) \leq\left\|I_{\varepsilon}^{\prime \prime}\left(z_{\mathbf{P}}\right)\right\| \sum_{i=1}^{K} \lambda_{i}^{2}\left\|\psi_{i}\right\|^{2} \leq C \sum_{i=1}^{K} \lambda_{i}^{2}\left\|\psi_{i}\right\|^{2}=o(1) .
$$

In the same way we obtain

$$
(I I) \leq\left\|I_{\varepsilon}^{\prime \prime}\left(z_{\mathbf{P}}\right)\right\| \sum_{i=1}^{K} \lambda_{i}^{2}\left\|\psi_{i}\right\|^{2} \leq C \sum_{i=1}^{K} \lambda_{i}^{2}\left\|\psi_{i}\right\|=o(1) .
$$

Furthermore, by making simple computations one finds

$$
\begin{aligned}
& (I)=\sum_{i=1}^{K} \lambda_{i}^{2}\left(\int_{\mathbb{R}^{3}}\left[\left|\nabla z_{P_{i}}\right|^{2}+z_{P_{i}}^{2}-p z_{P_{i}}^{p+1}\right] d x\right) \\
& +\sum_{i=1}^{K} \lambda_{i}^{2} \underbrace{\left(\int_{\mathbb{R}^{3}}[V(\varepsilon x)-1] z_{P_{i}}^{2} d x\right)}_{(A)} \\
& +2 \sum_{i \neq j} \lambda_{i} \lambda_{j} \underbrace{\left(\int_{\mathbb{R}^{3}}\left[\nabla z_{P_{i}} \nabla z_{P_{j}}+V(\varepsilon x) z_{P_{i}} z_{P_{j}}\right] d x\right)}_{(B)} \\
& +\underbrace{\varepsilon^{2} \int_{\mathbb{R}^{3}} \phi_{z_{\mathbf{P}}}\left(\sum_{i=1}^{K} \lambda_{i} z_{P_{i}}\right)^{2} d x}_{(C)}+\underbrace{2 \varepsilon^{2} \int_{\mathbb{R}^{3}} \widetilde{\phi} \cdot z_{\mathbf{P}}\left(\sum_{i=1}^{K} \lambda_{i} z_{P_{i}}\right) d x}_{(D)} \\
& -\underbrace{p \int_{\mathbb{R}^{3}}\left[\left|\sum_{i=1}^{K} z_{P_{i}}\right|^{p-1}\left(\sum_{i=1}^{K} \lambda_{i} z_{P_{i}}\right)^{2}-\sum_{i=1}^{K} \lambda_{i}^{2} z_{P_{i}}^{p+1}\right] d x}_{(E)}
\end{aligned}
$$

where $\widetilde{\phi}$ solves $-\Delta \widetilde{\phi}=\left(\sum_{i=1}^{K} \lambda_{i} z_{P_{i}}\right) z_{\mathbf{P}}$. 
Reasoning as in the proof of Proposition 3.2, we obtain that $(A)=o(1)$, $(B)=o(1),(C)=o(1),(D)=o(1)$. Moreover

$$
(E) \leq C\left(\lambda_{i}\right) \int_{\mathbb{R}^{3}}\left[\left|z_{\mathbf{P}}\right|^{p+1}-\sum_{i=1}^{K} z_{P_{i}}^{p+1}\right] d x .
$$

Then $(E)=o(1)$ as $\varepsilon \rightarrow 0$ (see Proposition 3.2). At the end

$$
I_{\varepsilon}^{\prime \prime}\left(z_{\mathbf{P}}\right)[u, u]=\sum_{i=1}^{K} \lambda_{i}^{2} I_{0}^{\prime \prime}\left(z_{P_{i}}\right)\left[z_{P_{i}}, z_{P_{i}}\right]+o(1) .
$$

Therefore, using Lemma 2.1 we have, for $\varepsilon$ small, that

$$
I_{\varepsilon}^{\prime \prime}\left(z_{\mathbf{P}}\right)[u, u] \leq(1-p) \sum_{i=1}^{K} \lambda_{i}^{2}\left\|z_{P_{i}}\right\|^{2}<-C_{1}<0 .
$$

So $I_{\varepsilon}^{\prime \prime}\left(z_{\mathbf{P}}\right)$ is negative definite on $A$. We now prove that $I_{\varepsilon}^{\prime \prime}\left(z_{\mathbf{P}}\right)$ is positive definite on $B$.

Choose an arbitrary $u \in B$. For simplicity, assume that $\|u\|=1$. We denote by $\hat{\phi}$ the solution of $-\Delta \hat{\phi}=z_{\mathbf{P}} u$. Since $z_{\mathbf{P}}$ and $u$ are bounded, it is easy to see that, for $\varepsilon$ small enough,

$$
\varepsilon^{2} \int_{\mathbb{R}^{3}}\left[\phi_{z_{\mathbf{P}}} u^{2}+2 \hat{\phi} z_{\mathbf{P}} u\right] d x=\int_{\mathbb{R}^{3}}[V(\varepsilon x)-1] u^{2} d x=o(1) .
$$

Then

$$
\begin{aligned}
I_{\varepsilon}^{\prime \prime}\left(z_{\mathbf{P}}\right)[u, u] & =\int_{\mathbb{R}^{3}}\left[|\nabla u|^{2}+V(\varepsilon x) u^{2}+\varepsilon^{2} \phi_{z_{\mathbf{P}}} u^{2}+2 \varepsilon^{2} \hat{\phi} z_{\mathbf{P}} u-p z_{\mathbf{P}}^{p-1} u^{2}\right] d x \\
& =\int_{\mathbb{R}^{3}}\left[|\nabla u|^{2}+u^{2}-p z_{\mathbf{P}}^{p-1} u^{2}\right] d x+o(1) .
\end{aligned}
$$

As done in Proposition 3.2 it can be proved that

$$
\int_{\mathbb{R}^{3}} z_{\mathbf{P}}^{p-1} u^{2} d x=\int_{\mathbb{R}^{3}} \sum_{i=1}^{K} z_{P_{i}}^{p-1} u^{2} d x+o(1) .
$$

Hence

$$
I_{\varepsilon}^{\prime \prime}\left(z_{\mathbf{P}}\right)[u, u]=\int_{\mathbb{R}^{3}}\left[|\nabla u|^{2}+u^{2}-p \sum_{i=1}^{K} z_{P_{i}}^{p-1} u^{2}\right] d x+o(1) .
$$

We need to estimate the integral in (3.5). In order to do this, we use the following technical result: 
Claim: for $\varepsilon$ small there exists $R \in\left(\varepsilon^{\frac{\theta}{2}}, \frac{1}{2} \varepsilon^{\theta}\right)$, with $\theta=\frac{2-\alpha}{\alpha+1}+\delta<0$, such that

$$
\sum_{i=1}^{K} \int_{R<\left|x-P_{i}\right|<R+1}\left[|\nabla u|^{2}+u^{2}\right] d x<4 \varepsilon^{-\theta}
$$

To prove this we remark that from $\|u\|=1$ it follows

$$
\sum_{i=1}^{K} \sum_{R \in\left(\varepsilon^{\frac{\theta}{2}}, \frac{1}{2} \varepsilon^{\theta}\right)} \int_{R<\left|x-P_{i}\right|<R+1}\left[|\nabla u|^{2}+u^{2}\right] d x \leq 1 \quad R \in \mathbb{N}
$$

Since, for $\varepsilon$ small, the above sum has more than $\frac{\varepsilon^{\theta}}{4}$ summands, then, it is always possible to choose $R \in \mathbb{N}, R \in\left(\varepsilon^{\frac{\theta}{2}}, \frac{1}{2} \varepsilon^{\theta}\right)$ such that the claim holds.

Let us fix $R$ such that (3.6) is satisfied and define the smooth cut-off functions $\chi_{i}: \mathbb{R} \rightarrow[0,1], i=1, \ldots, K$ by setting

$$
\chi_{i}(x):= \begin{cases}1 & \left|x-P_{i}\right|<R \\ 0 & \left|x-P_{i}\right|>R+1 \\ \left|\nabla \chi_{i}(x)\right| \leq 2 & \forall x \in \mathbb{R}^{3} .\end{cases}
$$

Define also

$$
\chi_{0}(x)=1-\sum_{i=1}^{K} \chi_{i}(x)
$$

Then we can decompose $u=\sum_{i=0}^{K} u_{i}$ where $u_{i}=u \chi_{i}$. From (3.6) it follows that for $i \neq j\left(u_{i}, u_{j}\right)=o(1)$. Thus

$$
1=\|u\|^{2}=\sum_{i=0}^{K}\left\|u_{i}\right\|^{2}+o(1) .
$$

Using again (3.6), we obtain that $\left(z_{P_{i}}, u_{j}\right)=o(1)$ for $i>0, i \neq j$. Since $u \in B$ we have $\left(z_{P_{i}}, u\right)=o(1)$. Then for $i=1, \ldots, K$

$$
\left(z_{P_{i}}, u\right)=\sum_{j=0}^{K}\left(z_{P_{i}}, u_{j}\right)=\left(z_{P_{i}}, u_{i}\right)+o(1) .
$$

Hence $\left(z_{P_{i}}, u_{i}\right)=o(1)$. Finally, for $i=1, \ldots, K$, since $u \perp \dot{z}_{i, j}$, reasoning as above, we find also $\left(\dot{z}_{i, j}, u_{l}\right)=o(1)$ for all $i, j, l$. 
By using the above properties and Lemma 2.1 we obtain

$$
\begin{aligned}
I_{\varepsilon}^{\prime \prime}\left(z_{\mathbf{P}}\right)[u, u] & =\int_{\mathbb{R}^{3}}\left[|\nabla u|^{2}+u^{2}-p \sum_{i=1}^{K} z_{P_{i}}^{p-1} u^{2}\right] d x+o(1) \\
& =\sum_{i=1}^{K} \int_{\mathbb{R}^{3}}\left[\left|\nabla u_{i}\right|^{2}+u_{i}^{2}-p z_{P_{i}}^{p-1} u_{i}^{2}\right] d x+\left\|u_{0}\right\|^{2}+o(1) \\
& \geq C \sum_{i=1}^{K}\left\|u_{i}\right\|^{2}+\left\|u_{0}\right\|^{2}+o(1) \\
& \geq C_{2}\left(\sum_{i=0}^{K}\left\|u_{i}\right\|^{2}\right)+o(1) \\
& \geq C_{2}>0 .
\end{aligned}
$$

With this estimates in hand we can now solve the auxiliary equation. Consider $z=z_{\mathbf{P}} \in \mathcal{Z}$ fixed, and define

$$
B_{\varepsilon}=\left\{u \in W:\|u\| \leq 2 \bar{C}\left\|I_{\varepsilon}^{\prime}(z)\right\|\right\}
$$

where $\bar{C}$ is the positive constant given by Proposition 3.3. So, the solutions of the auxiliary equations are fixed points of the map $S_{\varepsilon}: W \rightarrow W$

$$
S_{\varepsilon}(w)=w-\left[P I_{\varepsilon}^{\prime \prime}(z)\right]^{-1}\left[P I_{\varepsilon}^{\prime}(z+w)\right] .
$$

It is easy to check that $\left\|S_{\varepsilon}(0)\right\| \leq \bar{C}\left\|I_{\varepsilon}^{\prime}(z)\right\|$. We now compute the derivative of $S_{\varepsilon}$ :

$S_{\varepsilon}^{\prime}(w)[v]=v-\left[P I_{\varepsilon}^{\prime \prime}(z)\right]^{-1} P I_{\varepsilon}^{\prime \prime}(z+w)[v]=\left[P I_{\varepsilon}^{\prime \prime}(z)\right]^{-1}\left(P I_{\varepsilon}^{\prime \prime}(z)-P I_{\varepsilon}^{\prime \prime}(z+w)\right)[v]$.

Now observe that $I_{\varepsilon}^{\prime \prime}$ is uniformly continuous in bounded sets, so

$$
\left\|P I_{\varepsilon}^{\prime \prime}(z+w)-P I_{\varepsilon}^{\prime \prime}(z)\right\| \rightarrow 0 \quad(\varepsilon \rightarrow 0)
$$

uniformly in $z \in \mathcal{Z}$ and $w \in B_{\varepsilon}$ (recall Proposition 3.2).

This implies that $\left\|S_{\varepsilon}^{\prime}(w)\right\|=o(1)$ for any $w \in B_{\varepsilon}$. Therefore, $S_{\varepsilon}$ is a contraction and, by using the mean value theorem, $S_{\varepsilon}\left(B_{\varepsilon}\right) \subset B_{\varepsilon}$. We make use of the Banach contraction theorem to find a unique fixed point $w=w_{\varepsilon, z} \in B_{\varepsilon}$ of $S_{\varepsilon}$. Moreover one has

$$
\left\|w_{\varepsilon, z}\right\| \leq 2 \bar{C}\left\|I_{\varepsilon}^{\prime}(z)\right\| \leq C \varepsilon^{2}
$$




\section{The reduced functional}

In this section we will find a solution for the bifurcation equation among the set of solutions of the auxiliary equation, which is:

$$
\overline{\mathcal{Z}}=\left\{z+w_{\varepsilon, z}: z \in \mathcal{Z}, w_{\varepsilon, z} \text { solves (3.1)(a), and satisfies (3.7) }\right\} .
$$

By the Implicit Function Theorem it is easy to check that $\overline{\mathcal{Z}}$ is a $C^{1}$ manifold. Moreover, it is well-known (see [2], for example) that $\overline{\mathcal{Z}}$ is a natural constraint for $I_{\varepsilon}$ for $\varepsilon$ small. In other words, critical points of $\left.I_{\varepsilon}\right|_{\overline{\mathcal{Z}}}$ are solutions of the bifurcation equation (3.1) (b), and hence solutions of (2.2).

So, let us define the reduced functional as the restriction of the functional $I_{\varepsilon}$ to the natural constraint $\overline{\mathcal{Z}}$, namely $\Phi_{\varepsilon}: \Lambda_{\epsilon} \rightarrow \mathbb{R}, \Phi_{\varepsilon}(\mathbf{P})=$ $I_{\varepsilon}\left(z_{\mathbf{P}}+w_{\varepsilon, z_{\mathbf{P}}}\right)$, and we look for critical points of $\Phi_{\varepsilon}$. Using the information on $\left\|w_{\varepsilon, z_{\mathbf{P}}}\right\|$, we will be able to find an expansion of $\Phi_{\varepsilon}(\mathbf{P})$.

First of all, since $I_{\varepsilon}^{\prime \prime}$ maps bounded sets onto bounded sets, we have

$$
\Phi_{\varepsilon}(\mathbf{P})=I_{\varepsilon}\left(z_{\mathbf{P}}\right)+I_{\varepsilon}^{\prime}\left(z_{\mathbf{P}}\right)\left[w_{\varepsilon, z_{\mathbf{P}}}\right]+O\left(\left\|w_{\varepsilon, \mathbf{P}}\right\|^{2}\right) .
$$

Using Proposition 3.2 and (3.7) we deduce

$$
\Phi_{\varepsilon}(\mathbf{P})=I_{\varepsilon}\left(z_{\mathbf{P}}\right)+O\left(\varepsilon^{4}\right) .
$$

So we have to compute $I_{\varepsilon}\left(z_{\mathbf{P}}\right)$. Preliminary lemmas are in order.

Lemma 4.1. For $\beta=1,2$ and $F: \mathbb{R}^{3} \rightarrow \mathbb{R}$ such that $\left(1+|y|^{\beta+1}\right) F \in L^{1} \cap L^{\infty}$ set

$$
\Psi_{\beta}[F](x)=\int_{\mathbb{R}^{3}} \frac{1}{|x-y|^{\beta}} F(y) d y .
$$

Then there exist two positive constants $C=C(\beta, F)$ and $C^{\prime}=C^{\prime}(\beta, F)$ such that

$$
\left|\Psi_{\beta}[F]-\frac{C}{|x|^{\beta}}\right| \leq \frac{C^{\prime}}{|x|^{\beta+1}}, \quad \forall x \neq 0
$$

For a proof see [11]. Now, thanks to the the exponential decay of $U$ the following estimate holds (see Lemma 2.1 of [15]):

Lemma 4.2. For $\varepsilon$ sufficiently small and $\mathbf{P} \in \Lambda_{\varepsilon}$, we have

$$
\int_{\mathbb{R}^{3}} z_{P_{i}}^{p} z_{P_{j}} d x=(\eta+o(1)) e^{-\left|P_{1}-P_{2}\right|}
$$

where

$$
\eta=\int_{\mathbb{R}^{3}} U^{p}(x) e^{-x_{1}} d x>0
$$


We are now in position to find an expansion of $I_{\varepsilon}\left(z_{\mathbf{P}}\right)$.

Proposition 4.3. For any $\mathbf{P}=\left(P_{1}, \ldots, P_{K}\right) \in \Lambda_{\varepsilon}$ and $\varepsilon>0$ sufficiently small we have

$$
I_{\varepsilon}\left(z_{\mathbf{P}}\right)=C_{0}+\varepsilon^{2} C_{1}+C_{2} \sum_{i=1}^{K} V\left(\varepsilon P_{i}\right)+C_{3} \varepsilon^{2} \sum_{i \neq j} \frac{1}{\left|P_{i}-P_{j}\right|}+o\left(\varepsilon^{\frac{3 \alpha}{\alpha+1}-\delta}\right)
$$

where

$$
\begin{aligned}
& C_{0}=K \cdot\left(\frac{1}{2} \int_{\mathbb{R}^{3}}|\nabla U|^{2} d x-\frac{1}{p+1} \int_{\mathbb{R}^{3}} U^{p+1} d x\right), \\
& C_{1}=\frac{K}{4} \int_{\mathbb{R}^{3}} \frac{U^{2}(x) U^{2}(y)}{|x-y|} d x d y, \\
& C_{2}=\frac{1}{2} \int U^{2} d x,
\end{aligned}
$$

and $C_{3}$ is a positive constant given by Lemma 4.1, which depends only on $U$.

Proof. We compute

$$
\begin{aligned}
I_{\varepsilon}\left(z_{\mathbf{P}}\right)= & \sum_{i=1}^{K} I_{\varepsilon}\left(z_{P_{i}}\right)+\sum_{i \neq j} \int_{\mathbb{R}^{3}}\left[\nabla z_{P_{i}} \nabla z_{P_{j}}+V(\varepsilon x) z_{P_{i}} z_{P_{j}}\right] d x \\
& +\frac{\varepsilon^{2}}{4} \sum_{i \neq j} \int_{\mathbb{R}^{3}} \phi_{z_{P_{i}}} z_{P_{j}}^{2} d x+\frac{\varepsilon^{2}}{2} \sum_{l, i \neq j} \int_{\mathbb{R}^{3}} \phi_{i, j} z_{l}^{2} d x \\
& +\frac{\varepsilon^{2}}{4} \sum_{i \neq j} \int_{\mathbb{R}^{3}} \phi_{z_{\mathbf{P}}} z_{P_{i}} z_{P_{j}} d x-\frac{1}{p+1} \int_{\mathbb{R}^{3}}\left[\left|z_{\mathbf{P}}\right|^{p+1}-\sum_{i=1}^{K}\left|z_{P_{i}}\right|^{p+1}\right] d x \\
= & \sum_{i=1}^{K} I_{\varepsilon}\left(z_{P_{i}}\right)+\sum_{i \neq j} \int_{\mathbb{R}^{3}} z_{P_{i}}^{p} z_{P_{j}} d x+\frac{\varepsilon^{2}}{4} \sum_{i \neq j} \int_{\mathbb{R}^{3}} \phi_{z_{P_{i}}} z_{P_{j}}^{2} d x \\
& +\frac{\varepsilon^{2}}{2} \sum_{l, i \neq j} \int_{\mathbb{R}^{3}} \phi_{i, j} z_{l}^{2} d x+\frac{\varepsilon^{2}}{4} \sum_{i \neq j} \int_{\mathbb{R}^{3}} \phi_{z_{\mathbf{P}}} z_{P_{i}} z_{P_{j}} d x \\
& -\frac{1}{p+1} \int_{\mathbb{R}^{3}}\left[\left|z_{\mathbf{P}}\right|^{p+1}-\sum_{i=1}^{K}\left|z_{P_{i}}\right|^{p+1}\right] d x+o\left(\varepsilon^{\frac{3 \alpha}{\alpha+1}-\delta}\right) .
\end{aligned}
$$

Here $\phi_{i, j}$ are the solutions of $-\Delta \phi_{i, j}=z_{P_{i}} z_{P_{j}}, i \neq j$. Let us evaluate separately the various terms.

Claim: There holds:

$$
I_{\varepsilon}\left(z_{P_{i}}\right)=\widetilde{C}_{0}+\varepsilon^{2} \widetilde{C_{1}}+C_{2} V\left(\varepsilon P_{i}\right)+o\left(\varepsilon^{\frac{3 \alpha}{\alpha+1}-\delta}\right)
$$


where

$$
\begin{aligned}
\widetilde{C}_{0} & =\frac{1}{2} \int_{\mathbb{R}^{3}}|\nabla U|^{2} d x-\frac{1}{p+1} \int_{\mathbb{R}^{3}}|U|^{p+1} d x, \quad \widetilde{C_{1}}=\frac{1}{4} \int_{\mathbb{R}^{3}} \phi_{U} U^{2} d x, \\
C_{2} & =\frac{1}{2} \int_{\mathbb{R}^{3}} U^{2} d x .
\end{aligned}
$$

It suffices to estimate:

$$
\int_{\mathbb{R}^{3}}\left[V(\varepsilon x)-V\left(\varepsilon P_{i}\right)\right] U^{2}\left(x-P_{i}\right) d x .
$$

First, we split this integral expression in two terms

$$
\begin{aligned}
\int_{\mathbb{R}^{3}}\left[V(\varepsilon x)-V\left(\varepsilon P_{i}\right)\right] z_{P_{i}}^{2} d x= & \int_{\left|x-P_{i}\right|>\varepsilon^{-\tau}}\left[V(\varepsilon x)-V\left(\varepsilon P_{i}\right)\right] z_{P_{i}}^{2} d x \\
& +\int_{\left|x-P_{i}\right|<\varepsilon^{-\tau}}\left[V(\varepsilon x)-V\left(\varepsilon P_{i}\right)\right] z_{P_{i}}^{2} d x,
\end{aligned}
$$

for some positive constant $\tau$ to be determined. Since $V$ is bounded in $L^{\infty}$, we use the change $y=x-P_{i}$, and the exponential decay of $U$ to conclude

$$
\int_{\left|x-P_{i}\right|>\varepsilon^{-\tau}}\left[V(\varepsilon x)-V\left(\varepsilon P_{i}\right)\right] z_{P_{i}}^{2} d x \leq C \int_{|y|>\varepsilon^{-\tau}} U^{2}(y) d y=o\left(\varepsilon^{M}\right)
$$

for any positive $M$.

We use a Taylor expansion:

$$
\begin{aligned}
& \left|\int_{\left|x-P_{i}\right|<\varepsilon^{-\tau}}\left[V(\varepsilon x)-V\left(\varepsilon P_{i}\right)-\varepsilon \nabla V\left(\varepsilon P_{i}\right) \cdot\left(x-P_{i}\right)\right] z_{P_{i}}^{2}\right| \leq \\
& \quad \leq \frac{\varepsilon^{2}}{2} \max \left\{\left\|D^{2} V(\xi)\right\|:\left|\xi-\varepsilon P_{i}\right|<\varepsilon^{1-\tau}\right\} \int_{\mathbb{R}^{3}}\left|x-P_{i}\right|^{2} z_{P_{i}}^{2} d x .
\end{aligned}
$$

By using the radial symmetry of $U$,

$$
\int_{\left|x-P_{i}\right|<\varepsilon^{-\tau}} \nabla V\left(\varepsilon P_{i}\right) \cdot\left(x-P_{i}\right) U\left(x-P_{i}\right)^{2}=0 .
$$

So, it suffices to estimate $\left\|D^{2} V(\xi)\right\|$ for $\left|\xi-\varepsilon P_{i}\right|<\varepsilon^{1-\tau}$. First, observe that if $\tau<1$ and $\varepsilon$ is small enough, $\xi \in \mathcal{U}$.

Moreover, by the definition of $\Lambda_{\varepsilon}, V\left(\varepsilon P_{i}\right)=1+\left|g\left(\varepsilon P_{i}\right)\right|^{\alpha} \leq 1+\varepsilon^{\frac{3 \alpha}{\alpha+1}-\delta}$. From this and (2.4) we have that

$$
\left|V_{x_{i} x_{j}}\left(\varepsilon P_{i}\right)\right| \leq C|g(x)|^{\alpha-2} \leq C \varepsilon^{\frac{\alpha-2}{\alpha}\left(\frac{3 \alpha}{\alpha+1}-\delta\right)} .
$$


On the other hand, since $V \in C^{2, \gamma}$ (recall, $\left.\gamma=\min \{1, \alpha-2\}\right)$ :

$$
\left|V_{x_{i} x_{j}}(\xi)-V_{x_{i} x_{j}}\left(\varepsilon P_{i}\right)\right| \leq C \varepsilon^{\gamma(1-\tau)} \text {. }
$$

Therefore,

$$
\left|V_{x_{i} x_{j}}(\xi)\right| \leq C \varepsilon^{\min \left\{\frac{\alpha-2}{\alpha}\left(\frac{3 \alpha}{\alpha+1}-\delta\right), \gamma(1-\tau)\right\}} .
$$

By direct computation, $2+\frac{\alpha-2}{\alpha}\left(\frac{3 \alpha}{\alpha+1}-\delta\right)>\frac{3 \alpha}{\alpha+1}-\delta$. Moreover, $2+1=$ $3>\frac{3 \alpha}{\alpha+1}$ and $2+\alpha-2=\alpha>\frac{3 \alpha}{\alpha+1}$. Then, we can choose $\tau>0$ small enough such that $2+\gamma(1-\tau)>\frac{3 \alpha}{\alpha+1}-\delta$. This concludes the proof of the claim.

We now continue the estimates of the remaining terms. From Lemma 4.2

$$
\int_{\mathbb{R}^{3}} z_{P_{i}}^{p} z_{P_{j}} d x=(\eta+o(1)) e^{-\left|P_{i}-P_{j}\right|}=o\left(\varepsilon^{M}\right)
$$

for any $M>0$. Now, by using the notations of Lemma 4.1, we have

$$
\phi_{z_{P_{i}}}=\frac{1}{4 \pi} \Psi_{1}\left[U^{2}\right]\left(x-P_{i}\right) .
$$

If $i \neq j$, by $(4.2)$

$$
\begin{aligned}
& \int_{\mathbb{R}^{3}} \phi_{z_{P_{i}}} z_{P_{j}}^{2} d x=\frac{1}{4 \pi} \int_{\mathbb{R}^{3}} \Psi_{1}\left[U^{2}\right]\left(x-P_{i}\right) U^{2}\left(x-P_{j}\right) d x \\
& \quad=C_{3} \int_{\mathbb{R}^{3}} \frac{1}{\left|x-P_{i}\right|} U^{2}\left(x-P_{j}\right) d x+O(1) \int_{\mathbb{R}^{3}} \frac{1}{\left|y+P_{j}-P_{i}\right|^{2}} U^{2}(y) d x \\
& \quad=C_{3} \Psi_{1}\left(U^{2}\right)\left|P_{i}-P_{j}\right|+O(1)\left|P_{i}-P_{j}\right|^{-2} .
\end{aligned}
$$

From the definition of $\Lambda_{\varepsilon}$ and since $\alpha>2,\left|P_{i}-P_{j}\right|^{-2}=o\left(\varepsilon^{\frac{3 \alpha}{\alpha+1}-\delta}\right)$. Furthermore

$$
\int_{\mathbb{R}^{3}} \phi_{z_{\mathbf{P}}} z_{P_{i}} z_{P_{j}} d x \leq C \int_{\mathbb{R}^{3}} z_{P_{i}} z_{P_{j}} d x=o\left(\varepsilon^{M}\right)(i \neq j) .
$$

and, consequently,

$$
\int_{\mathbb{R}^{3}} \phi_{i, j} z_{P_{i}}^{2} d x=-\int_{\mathbb{R}^{3}} \phi_{i, j} \Delta \phi_{z_{P_{i}}}=-\int_{\mathbb{R}^{3}} \Delta \phi_{i, j} \phi_{z_{P_{i}}}=\int_{\mathbb{R}^{3}} \phi_{z_{P_{i}}} z_{P_{i}} z_{P_{j}} d x=o\left(\varepsilon^{M}\right)
$$

for any $M>0$. Since $\mathbf{P} \in \Lambda_{\varepsilon}$, we have that for $i \neq j$

$$
\frac{\varepsilon^{2}}{4} \int_{\mathbb{R}^{3}} \phi_{z_{P_{i}}} z_{P_{j}}^{2} d x=C_{3} \frac{\varepsilon^{2}}{\left|P_{i}-P_{j}\right|}+\varepsilon^{2} O\left(\left|P_{i}-P_{j}\right|^{-2}\right)=C_{3} \frac{\varepsilon^{2}}{\left|P_{i}-P_{j}\right|}+o\left(\varepsilon^{\frac{3 \alpha}{\alpha+1}-\delta}\right) .
$$

Finally, arguing as in Proposition 3.2, we obtain

$$
\int_{\mathbb{R}^{3}}\left[\left|z_{\mathbf{P}}\right|^{p+1}-\sum_{i=1}^{K}\left|z_{P_{i}}\right|^{p+1}\right] d x=o\left(\varepsilon^{M}\right)
$$

for any $M>0$. All previous estimates imply the expansion (4.3). 
From (4.1) and (4.3) we have the following expansion for the reduced functional

$$
\Phi_{\varepsilon}(\mathbf{P})=C_{0}+\varepsilon^{2} C_{1}+C_{2} \sum_{i=1}^{K} V\left(\varepsilon P_{i}\right)+C_{3} \varepsilon^{2} \sum_{i \neq j} \frac{1}{\left|P_{i}-P_{j}\right|}+o\left(\varepsilon^{\frac{3 \alpha}{\alpha+1}-\delta}\right) .
$$

Proposition 4.4. For $\varepsilon$ sufficiently small, the following minimization problem

$$
\min \left\{\Phi_{\varepsilon}(\mathbf{P}): \mathbf{P} \in \Lambda_{\varepsilon}\right\}
$$

has a solution $\mathbf{P}_{\varepsilon} \in \Lambda_{\varepsilon}$.

Proof. Since $\Phi_{\varepsilon}(\mathbf{P})$ is continuous in $\mathbf{P}$ in a compact set, the minimization problem has a solution. Let $\Phi_{\varepsilon}\left(\mathbf{P}^{\varepsilon}\right)$ be the minimum of $\Phi_{\varepsilon}$ where $\mathbf{P}^{\varepsilon}$ is in the closure of the set $\Lambda_{\varepsilon}$. We prove by energy comparison that $\mathbf{P}^{\varepsilon}$ is not on the boundary of $\Lambda_{\varepsilon}$. In order to do this, first we obtain an upper bound for $\Phi_{\varepsilon}\left(\mathbf{P}^{\varepsilon}\right)$. Let us choose

$$
P_{j}^{0}=\varepsilon^{\frac{2-\alpha}{\alpha+1}} X_{j}
$$

where $X_{j}, j=1, \ldots, K$ are the $K$ vortices of $K$-polygon centered at 0 with $\left|X_{i}-X_{j}\right|=1, i \neq j$.

Then for $\varepsilon$ small it is clear that $\varepsilon P_{j}^{0} \in \mathcal{U}$. Moreover

$$
\left|P_{i}^{0}-P_{j}^{0}\right|=\varepsilon^{\frac{2-\alpha}{\alpha+1}}\left|X_{i}-X_{j}\right|
$$

and

$$
V\left(\varepsilon P_{j}^{0}\right) \leq 1+C\left|\varepsilon P_{j}^{0}\right|^{\alpha} \leq 1+C \varepsilon^{\frac{3 \alpha}{\alpha+1}} .
$$

Therefore, $\mathbf{P}^{\mathbf{0}}=\left(P_{1}^{0}, \ldots, P_{K}^{0}\right) \in \Lambda_{\varepsilon}$. Hence by (4.8) we obtain

$$
\Phi_{\varepsilon}\left(\mathbf{P}^{\varepsilon}\right)=\min _{\mathbf{P} \in \Lambda_{\varepsilon}} \Phi_{\varepsilon}(\mathbf{P}) \leq \Phi_{\varepsilon}\left(\mathbf{P}^{\mathbf{0}}\right) \leq C_{0}+\varepsilon^{2} C_{1}+K C_{2}+C_{3} \varepsilon^{\frac{3 \alpha}{\alpha+1}} .
$$

If now $\mathbf{P}^{\varepsilon}$ is such that $\left|P_{i}^{\varepsilon}-P_{j}^{\varepsilon}\right|=\varepsilon^{\frac{2-\alpha}{\alpha+1}+\delta}$ for some $i \neq j$, then

$$
\Phi_{\varepsilon}\left(\mathbf{P}^{\varepsilon}\right) \geq C_{0}+\varepsilon^{2} C_{1}+K C_{2}+C_{3} \varepsilon^{\frac{3 \alpha}{\alpha+1}-\delta} .
$$

If, instead, $\mathbf{P}^{\varepsilon}$ is such that $V\left(\varepsilon P_{i}^{\varepsilon}\right)=1+\varepsilon^{\frac{3 \alpha}{\alpha+1}-\delta}$ for some $i$, then

$$
\Phi_{\varepsilon}\left(\mathbf{P}^{\varepsilon}\right) \geq C_{0}+\varepsilon^{2} C_{1}+K C_{2}+C_{2} \varepsilon^{\frac{3 \alpha}{\alpha+1}-\delta} .
$$

But both (4.11) and (4.12) are in contradiction with (4.10).

We remark that we have not considered the case $\varepsilon \mathbf{P}^{\varepsilon} \in \partial \mathcal{U}$, because this would be in contradiction with $V\left(\varepsilon P_{j}^{\varepsilon}\right) \leq 1+\varepsilon^{\frac{3 \alpha}{\alpha+1}-\delta}$ for $\varepsilon$ small.

Acknowledgement: Part of this work has been carried out while G. Vaira was visiting the Department of Análisis Matemático in Granada. She would like to thank the members of the Department for their hospitality. 


\section{References}

[1] Ambrosetti, A., Badiale, M. and Cingolani, S.: Semiclassical states of nonlinear Schödinger equations. Arch. Rational Mech. Anal. 140 (1997), no. $3,285-300$.

[2] Ambrosetti, A. And Malchiodi, A.: Perturbation methods and semilinear elliptic problems on $\mathbb{R}^{n}$. Progress in Mathematics 240. Birkhäuser Verlag, Basel, 2006.

[3] Ambrosetti, A. And Ruiz, D.: Multiple bound states for the Schrödinger-Poisson problem. Commun. Contemp. Math. 10 (2008), no. 3, 391-404.

[4] Azzollini, A. And Pomponio, A.: Ground state solutions for the nonlinear Schrödinger-Maxwell equations. J. Math. Anal. Appl. 345 (2008), no. 1, 90-108.

[5] Benci, V. and Fortunato, D.: An eigenvalue problem for the Schrödinger-Maxwell equations. Topol. Methods Nonlinear Anal. 11 (1998), no. 2, 283-293.

[6] Bokanowski, O. And Mauser, N. J.: Local approximation for the Hartree-Fock exchange potential: a deformation approach. Math. Models Methods Appl. Sci. 9 (1999), no. 6, 941-961.

[7] Bokanowski, O., López, J. L. And Soler, J.: On an exchange interaction model for quantum transport; the Schrödinger-Poisson-Slater term. Math. Models Methods Appl. Sci. 13 (2003), no. 10, 1397-1412.

[8] Cornean, H., Hoke, K., Neidhardt, H., Racec, P. N. and ReHBERG, J.: A Kohn-Sham system at zero temperature. J. Phys. A $4 \mathbf{1}$ (2008), no. 38, 385304, 21pp.

[9] D'Aprile, T. And Mugnai, D.: Solitary waves for nonlinear KleinGordon-Maxwell and Schrödinger-Maxwell equations. Proc. Roy. Soc. Edinburgh Sect. A 134 (2004), no. 5, 893-906.

[10] D'Aprile, T. And Mugnai, D.: Non-existence results for the coupled KleinGordon-Maxwell equations. Adv. Nonlinear Stud. 4 (2004), no. 3, 307-322.

[11] D'Aprile, T. AND WeI, J.: Standing waves in the Maxwell-Schrödinger equation and an optimal configuration problem. Calc. Var. Partial Differential Equations 25 (2006), no. 1, 105-137.

[12] D'Aprile, T. AND Wei, J.: Clustered solutions around harmonic centers to a coupled elliptic system. Ann. Inst. H. Poincaré Anal. Non Linéaire $\mathbf{2 4}$ (2007), no. 4, 605-628.

[13] Del Pino, M. and Felmer, P.: Multi-peak bound states for nonlinear Schrödinger equations. Ann. Inst. H. Poincaré Anal. Non Linéaire 15 (1998), no. 2, 127-149.

[14] Gui, C.: Existence of multi-bump solutions for nonlinear Schrödinger equations via variational methods. Comm. Partial Differential Equations 21 (1996), no. 5-6, 787-820. 
[15] Kang, X. AND WeI, J.: On interacting bumps of semi-classical states of nonlinear Schrödinger equations. Adv. Differential Equations 5 (2000), no. 7-9, 899-928.

[16] KIKUCHI, H.: On the existence of a solution for elliptic system related to the Maxwell-Schrödinger equations. Nonlinear Anal. 67 (2007), no. 5, $1445-1456$.

[17] KikUCHI, H.: Existence and orbital stability of standing waves for nonlinear Schrödinger equations via the variational method. Doctoral Thesis.

[18] Kwong, M. K.: Uniqueness of positive solutions of $\Delta u-u+u^{p}=0$ in $\mathbb{R}^{N}$. Arch. Rational Mech. Anal. 105 (1989), no. 3, 243-266.

[19] IAnni, I. AND Vaira, G.: On concentration of positive bound states for the Schrödinger-Poisson problem with potentials. Adv. Nonlinear Stud. 8 (2008), no. 3, 573-595.

[20] Li, Y.: On a singularly perturbed elliptic equation. Adv. Differential Equations 2 (1997), no. 6, 955-980.

[21] Mauser, N. J.: The Schrödinger-Poisson-Xo equation. Appl. Math. Lett. 14 (2001), no. 6, 759-763.

[22] Pisani, L. and Siciliano, G.: Neumann condition in the SchrödingerMaxwell system. Topol. Methods Nonlinear Anal. 29 (2007), no. 2, 251-264.

[23] Ruiz, D.: The Schrödinger-Poisson equation under the effect of a nonlinear local term. J. Funct. Anal. 237 (2006), no. 2, 655-674.

[24] Slater, J. C.: A simplification of the Hartree-Fock method. Phys. Review 81 (1951), 385-390.

[25] SÁnchez, O. And Soler, J.: Long-time dynamics of the SchrödingerPoisson-Slater system. J. Statistical Physics 114 (2004), 179-204.

[26] Zhao, L. And Zhao, F.: On the existence of solutions for the SchrödingerPoisson equations. J. Math. Anal. Appl. 346 (2008), no. 1, 155-169.

[27] Wang, Z. And Zhou, H. S.: Positive solution for a nonlinear stationary Schrödinger-Poisson system in $\mathbb{R}^{3}$. Discrete Contin. Dyn. Syst. 18 (2007), no. 4, 809-816.

Recibido: 28 de abril de 2009

David Ruiz

Departamento de Análisis Matemático

University of Granada

18071 Granada, Spain daruiz@ugr.es

Giusi Vaira SISSA

via Bonomea 265

34136 Trieste, Italy

vaira@sissa.it 